\title{
Determination of the South Atlantic Anomaly from DAMPE data
}

\author{
Wei Jiang ${ }^{* a b}$, Xiang $\mathbf{L i}^{a}$, Jingjing Zang ${ }^{a}$, Chuan Yue ${ }^{a c}$ and Yuanpeng Wang ${ }^{a c}$ on \\ behalf of the DAMPE collaboration \\ ${ }^{a}$ Key Laboratory of Dark Matter and Space Astronomy, Purple Mountain Observatory, Chinese \\ Academy of Sciences, Nanjing 210008, China \\ ${ }^{b}$ School of Astronomy and Space Science, University of Science and Technology of China, Hefei, \\ 230026, China \\ ${ }^{c}$ University of Chinese Academy of Sciences, Beijing, 100012, China \\ E-mail: jiangwei@pmo.ac.cn
}

The DArk Matter Particle Explorer (DAMPE) can operate properly while crossing the South Atlantic Anomaly (SAA). Due to the high flux of cosmic rays in the SAA, the collected data within SAA are not used for most scientific analysis. Based on the data from DAMPE, we improve the definition of SAA with more precise determination of the boundary. We found that about $30 \%$ time in the pre-defined SAA period can be saved for normal data taking comparing with a typical polygon defined by the space weather forecast before launch.

35th International Cosmic Ray Conference - ICRC2017

10-20 July, 2017

Bexco, Busan, Korea

\footnotetext{
${ }^{*}$ Speaker.
} 


\section{Introduction}

The DArk Matter Particle Explorer (DAMPE)[1,2] was successfully launched on December 17th, 2015 from the Jiuquan Satellite Launch Center. DAMPE measures cosmic rays and gammarays in a very wide energy range for the study of high energy astrophysics as well as the nature of dark matter particles. Due to the non-concentricity of the Earth and its magnetic dipole, the Van Allen radiation belt dips down to $200 \mathrm{~km}$ in altitude in the South Atlantic Anomaly (SAA) region. The fluxes of trapped proton and electron in the radiation belt can reach two orders of magnitude larger than other areas [3, 4], which is believed to be an essential reason of failures of some satellites. DAMPE is operating around sun-synchronous orbit at the altitude of $\sim 500 \mathrm{~km}$ with an inclination of $\sim 97^{\circ}$. And it travels through the SAA about 7 times a day. The energy of trapped proton in the SAA is extremely low relative to DAMPE's sensitive energy range. Most part of them is blocked by shield of satellite. The simulations and ground beam tests show that the electronic components of DAMPE survived under such environment of high-energy particles without obvious performance degradation and life lost $[6,7]$. In this case, DAMPE keeps normal operation in SAA, where the intensive particle hits will dramatically affect the electronic readouts. So even though DAMPE can operate normally in the SAA, the data acquired in this region cannot be used for most scientific analyses and the events need to be tagged. Thus we use the recorded data of DAMPE to develop a method to precisely determine the boundary of the SAA.

\section{SAA determination}

Before launch, the SAA was already measured by many experiments $[3,4,5$, e.g.]. The space weather forecast made by the Space Environment Prediction Center, National Space Center, Chinese Academy of Sciences using AP-9[8] has already briefly defined the general map of the SAA region for the requirement of the whole project of DAMPE, seen in Fig.2. But such a kind of definition is not suitable for data analysis, because it is just a rough and conservative estimation as a result that too much observed time will be wasted when we analyze the data. During the operation of DAMPE, we found that the trigger rate with a low threshold of the top layers can directly reflect the particle flux in space, thereby providing a good and simple way to identify the SAA region. This trigger rate is too high to generate every event, and it is recorded as the count of the rate for each 4 seconds by a counter and transmitted to the ground as the housekeeping data. As is shown in Fig.1, the trigger rate has a periodic trend of high rate at polar and low rate near equator, however, it goes a sharp rise between about 20 degrees and 40 degrees southern latitude. These typical rises are defined as SAA periods, identified by the clear inflection points of the trigger rate. Therefore, we fit these inflection points of the trigger rate over time to determine when the satellite passes in and out the SAA. Thus the region of the SAA is defined as the positions where the satellite is during these periods, hence the detected data and live time between when the satellite enter and exit the SAA can be excluded for data analysis. 


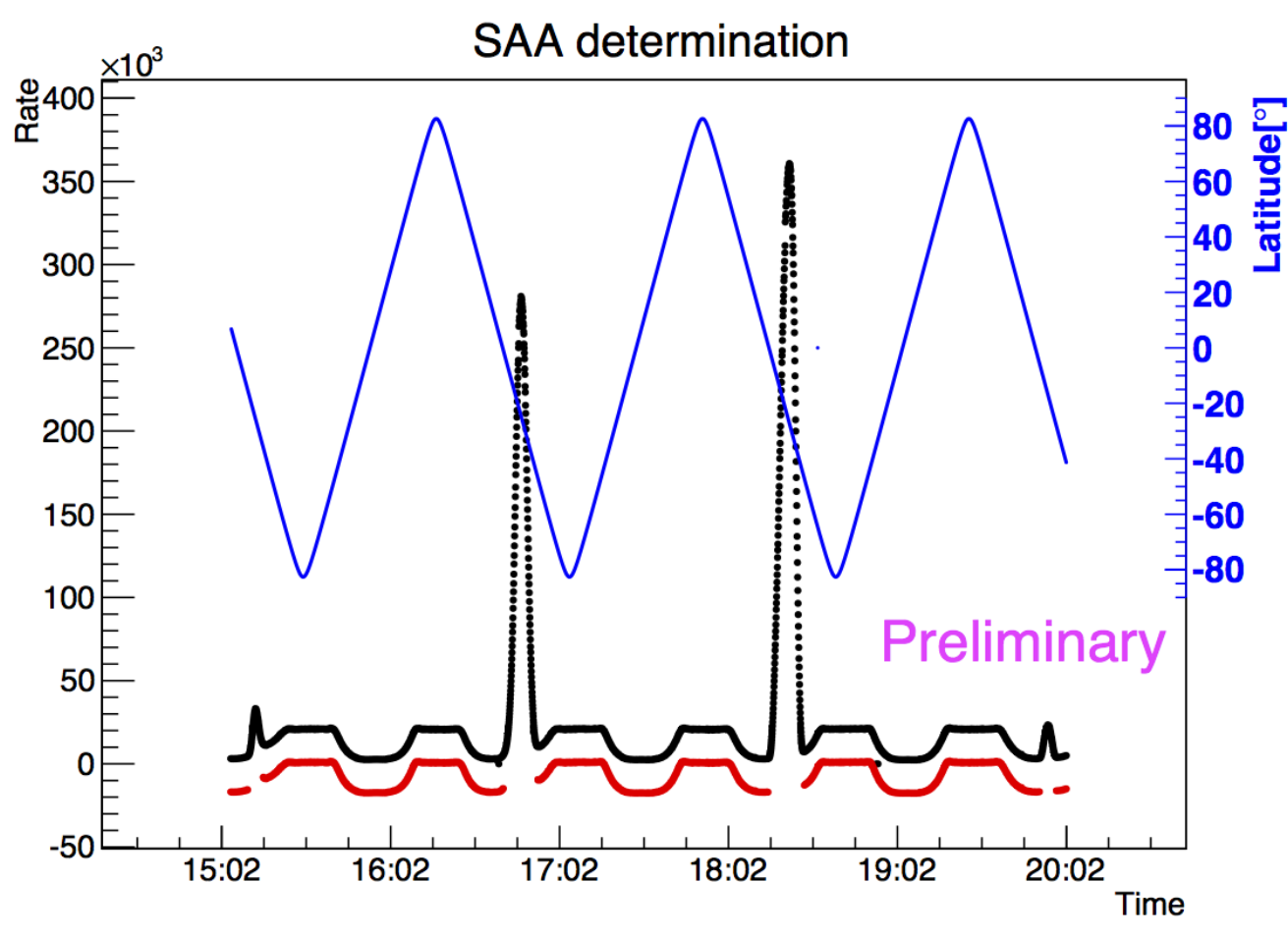

Figure 1: The trigger rate during a period when the satellite crosses the SAA 4 times. Black line is the rate before SAA rejection. As the blue line is labeled by latitude, it is clear that top platforms are polar regions and bottoms are equatorial regions. Red line is the rate after SAA rejection. To make a comparison, the red line is shifted down 20000 manually.

\section{SAA map}

The map of this trigger rate is plot in Fig.2, in which the trigger rate shows a sharp rise in the SAA region. From this map, we find that the previous determined SAA region is much smaller than that predicted by the space weather forecast with AP-9. Thus it obviously improve the efficiency for data analysis, i.e. about $30 \%$ of the AP-9-defined SAA period is saved. Some spatial experiments $[9,10]$ show that the location of the maximum particle density in SAA drifts at a rate of about $0.4^{\circ} \cdot y r^{-1}$ at the altitude close to DAMPE, the size and particle fluxes of the SAA vary with the solar cycle and geomagnetic activities as well. Since DAMPE doesn't have so precise measurement to the SAA, the determined SAA by DAMPE should not change a lot at a time-scale of month consequently. During a month, we can get $\sim 400$ boundary points of the determined SAA, thus we can fit the boundary for each month to monitor its variation. Fig.3 gives the comparison among the determined SAA region in the first 3 months in 2016, in which the boundary of the determined SAA varies little. It's the result of the stability of both this determination method and the SAA itself. As is applied, the method can update the SAA in real time, so the effect of the variation of the SAA is minimized. 


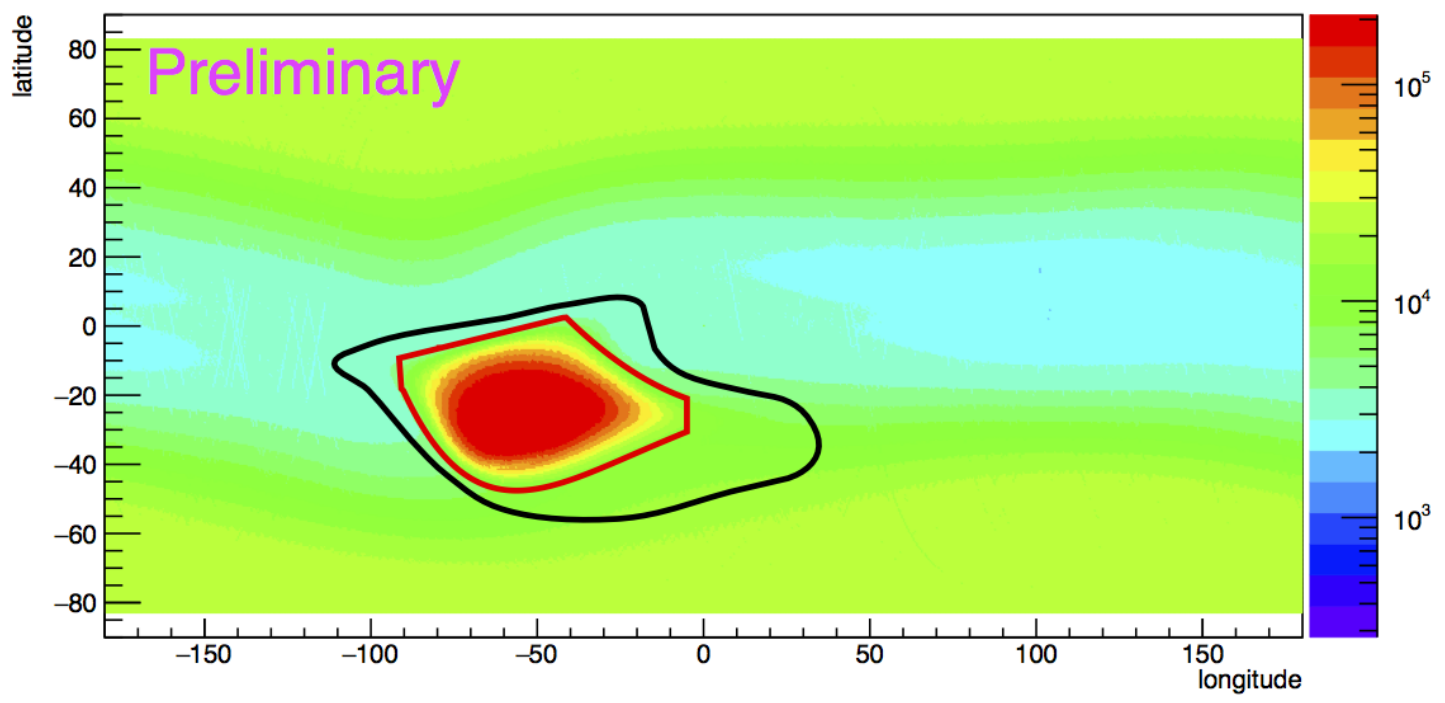

Figure 2: The trigger rate variation with geographic latitude and longitude. The red solid line is labeled as the SAA boundary fitted by the data from Jan. 1st to Dec. 31st, 2016. The black solid line is the contour about $1 \mathrm{~cm}^{-2} \mathrm{~s}^{-1}$ for trapped proton flux above $10 \mathrm{MeV}$ predicted by the AP-9[8].

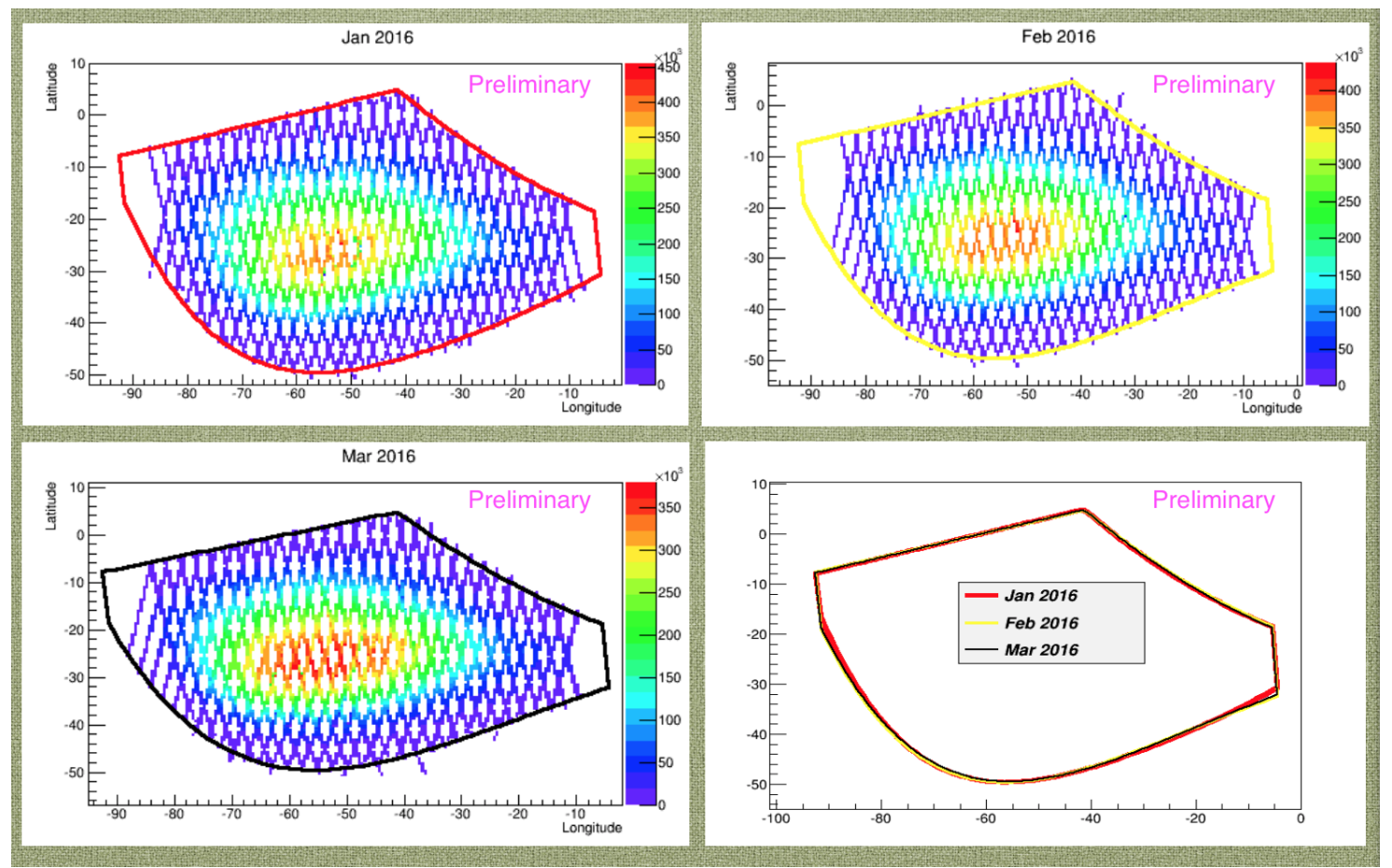

Figure 3: Comparison of the determinate SAA region among 3 months. The polygons are the fitted boundaries. It can be found that the boundary changes little over time. 


\section{Summary}

This method for identifying the SAA region is directly based on the cosmic-ray flux in space. By applying it, we can dynamically discard the "bad" data in the SAA region and keep as many good events as possible, thereby improving the efficiency for analysis. As time goes on in the future, based on this method, the variations of SAA can potentially be studied.

\section{Acknowladgement}

The DAMPE mission is funded by the strategic priority science and technology projects in space science of Chinese Academy of Sciences. In China the data analysis was supported in part by the National Key Research and Development Program of China (No. 2016YFA0400200), National Basic Research Program of China (No. 2013CB837000), NSFC (Nos. 11525313 and 11622327), the 100 Talents Program of Chinese Academy of Sciences, the Astronomy Joint Grant (U1631111), and Strategic Priority Research Program of the CAS "Multi-waveband Gravitational Wave Universe" (Grant No. XDB23040000). In Europe the activities and the data analysis are supported by the Swiss National Science Foundation (SNSF), Switzerland; the National Institute for Nuclear Physics (INFN), Italy.

\section{References}

[1] J. Chang, Chin. J. Spac. Sci., 34 (2014) 550.

[2] J. Chang et al. [DAMPE collaboration], submitted to Astroparticle Physics (arXiv: 1706.08453).

[3] A. A. Abdo, et al. [Fermi-LAT collaboration], Astropart. Phys., 32 (2009) 193-219.

[4] M. Ackermann, et al. [Fermi-LAT collaboration], Astrophysics. J. Supp., 203 (2012) 4.

[5] M. Casolino, et al., cosp 37(2008) 466C.

[6] S. S. Gao, C. Q. Feng, D. Jiang, S. B. Liu, Z. G. Zhang, K. Xi, \& Q. An. Nucl. Sci. Tech. 25 (2014) 010402.

[7] F. Zhang, et al., Chin. Phys. C., 40 (2016) 116101.

[8] G. P. Ginet, et al. Space Science Reviews, 179 (2013) 579-615.

[9] F. Fürst, et al., Earth and Planetary Science Letters 281 (2009)125.

[10] R. Campana, et al. Experimental Astronomy, 37(2014) 599-613. 